\title{
ULTRASEPARATING FUNCTION SPACES AND OPERATING FUNCTIONS FOR THE REAL PART OF A FUNCTION ALGEBRA
}

\author{
EGGERT BRIEM
}

(Communicated by John B. Conway)

\begin{abstract}
We show how a local version of a result due to A. Bernard on ultraseparating Banach function spaces can be used to give a short proof of the theorem stating that only affine functions operate on the real part of a function algebra.
\end{abstract}

The paper [5] by K. de Leeuw and Y. Katznelson contains the following generalization of the Stone-Weierstrass Theorem:

Let $X$ be a compact Hausdorff space and $B$ a subspace of $C_{\mathbf{R}}(X)$, which separates the points of $X$ and contains the constant functions. If there is a continuous non-affine function $h$ defined on a subinterval $I$ of $\mathbf{R}$ which operates on $B$, i.e., $h \circ b \in B$ if $b \in B$ and $h \circ b$ is defined, then $B$ is dense in $C_{\mathbf{R}}(X)$.

At the same time J. Wermer [8] proved the following theorem:

Let $A$ be a function algebra on a compact Hausdorff space $X$. Then if $\operatorname{Re} A$ is an algebra, $A=C(X)$.

Here, $\operatorname{Re} A$ denotes the space of the real parts of functions in $A$. Another formulation of Wermer's result is that if the function $t \mapsto t^{2}$ operates on $\operatorname{Re} A$, then $A=C(X)$. These two results lead to the following conjecture:

Let $A$ be a function algebra on the compact Hausdorff space $X$. If there is a non-affine function defined on a subinterval of $\mathbf{R}$ which operates on $\operatorname{Re} A$, then $A=C(X)$.

(Here, it is not necessary to assume that the operating function is continuous because a function which operates on the real part of a function algebra is automatically continuous [6].)

If one tries to apply directly the theorem of de Leeuw and Katznelson one only finds that $\operatorname{Re} A$ is a dense subspace of $C_{\mathbf{R}}(X)$, i.e., that $A$ is a Dirichlet algebra. Thus, more is needed to prove the conjecture.

In [1] A. Bernard introduced a powerful method to attack the conjecture and related problems: Let $\beta(\mathbf{N} \times X)$ denote the Stone-Čech compactification of $\mathbf{N} \times X$, and let $l^{\infty}(\mathbf{N}, C(X))$ denote the space of all bounded sequences

Received by the editors August 31, 1988.

1980 Mathematics Subject Classification (1985 Revision). Primary 46B15, 46J10. 
of continuous functions on $X$. This space can be identified in a natural way with $C(\beta(\mathbf{N} \times X))$, the space of all continuous complex-valued functions on $\beta(\mathbf{N} \times X)$. Now, if $A$ is a function algebra on $X$, one can introduce a norm on $\operatorname{Re} A$ in the following manner:

$$
N(u)=\inf \{\|a\|: a \in A \text { and } \operatorname{Re} a=u\} \quad \text { for all } u \in \operatorname{Re} A
$$

where $\|\cdot\|$ denotes the supremum norm. This norm dominates the supremum norm and turns $\operatorname{Re} A$ into a Banach space. The space $l^{\infty}(\mathbf{N}, \operatorname{Re} A)$ of all $N$-bounded sequences of elements from $\operatorname{Re} A$ satisfies the inclusion $l^{\infty}(\mathbf{N}, \operatorname{Re} A) \subseteq l^{\infty}\left(\mathbf{N}, C_{\mathbf{R}}(X)\right)=C_{\mathbf{R}}(\beta(\mathbf{N} \times X))$. It turns out that if $\operatorname{Re} A$ is a Dirichlet algebra then $l^{\infty}(\mathbf{N}, \operatorname{Re} A)$ separates the points of $\beta(\mathbf{N} \times X)$, (cf. [1]). From the theorem of de Leeuw and Katznelson it thus follows that if there is a non-affine function which operates on $l^{\infty}(\mathbf{N}, \operatorname{Re} A)$ then $l^{\infty}(\mathbf{N}, \operatorname{Re} A)$ is dense in $C_{\mathbf{R}}(\beta(\mathbf{N} \times X))$. This has some interesting consequences, as the next theorem, which is due to Bernard [1], shows.

Theorem. Let $E$ be a subspace of $C(X)$ which is a Banach space in a norm that dominates the supremum norm. Then, if $l^{\infty}(\mathbf{N}, E)$ is dense in $C(\beta(\mathbf{N} \times X))$, it follows that $E=C(X)$.

This theorem holds for the real as well as the complex case. Thus one concludes that if $l^{\infty}(\mathbf{N}, \operatorname{Re} A)$ is dense in $C_{\mathbf{R}}(\beta(\mathbf{N} \times X))$, then $\operatorname{Re} A=C_{\mathbf{R}}(X)$, and hence $A=C(X)$ by a well known theorem of Bishop, Hoffman and Wermer.

In general it is not clear that a function which operates on $\operatorname{Re} A$ also operates on $l^{\infty}(\mathbf{N}, \operatorname{Re} A)$. This led Bernard to introduce the following concept:

$A$ real-valued function $h$, defined on an open subinterval of $\mathbf{R}$ containing 0 , operates boundedly on $\operatorname{Re} A$ if there exist numbers $\alpha>0$ and $M>0$ such that $N(h \circ u)<M$ whenever $u \in \operatorname{Re} A$ and $N(u)<\alpha$.

As an example, take the function $t \mapsto t^{2}$. If this function operates on $\operatorname{Re} A$ then it operates boundedly on $\operatorname{Re} A$ (cf. [1]). If $h$ operates boundedly on $\operatorname{Re} A$ then $h \circ\left\{u_{n}\right\} \in l^{\infty}(\mathbf{N}, \operatorname{Re} A)$ whenever $\left\{u_{n}\right\} \in l^{\infty}(\mathbf{N}, \operatorname{Re} A)$ and $N\left(u_{n}\right)<\alpha$ for all $n$. The proof of the theorem of de Leeuw and Katznelson shows that if $h$ is also non-affine, then it follows that $l^{\infty}(\mathbf{N}, \operatorname{Re} A)$ is dense in $C_{\mathbf{R}}(\beta(\mathbf{N} \times X))$.

Using the Baire Category Theorem in a clever way S. Sidney [6] succeeded in proving the conjecture without the condition that the operating function should operate boundedly. However, he had to add another condition; the operating function must not be affine on any subinterval of the one on which it is defined. The finishing touch is due to O. Hatori [3], who proved the conjecture for the remaining case.

Recently O. Hatori [4] has given a local version of the theorem of Bernard stated above. In this note we show how a variant of Hatori's local version can be used to give a simple proof of the above conjecture. 


\section{RESULTS AND APPLICATIONS}

Let $X$ be a compact Hausdorff space. A subspace $B$ of $C(X)$ which separates the points of $X$, contains the constant functions and is complete in a norm which dominates the supremum norm is called a Banach function space on $X$. Bernard calls such a space ultraseparating on $X$ if $l^{\infty}(\mathbf{N}, B)$ separates the points of the Stone-Čech compactification $\beta(\mathbf{N} \times X)$ of $\mathbf{N} \times X$. As mentioned earlier, if $A$ is a Dirichlet algebra on $X$, then $\operatorname{Re} A$ is ultraseparating (see [1]).

If $f \in C(X)$ we let $\{f\}$ denote the element $\left\{f_{n}\right\} \in l^{\infty}(\mathbf{N}, C(X))$ where $f_{n}=f$ for all $n$. For $x \in X$ and $f \in C(X)$ we let $\mathscr{F}_{x}=\mathscr{F}_{x}(f)$ denote the set of constancy for $\{f\}$ given by

$$
\mathscr{F}_{x}=\{\xi \in \beta(\mathbf{N} \times X):\{f\}(\xi)=f(x)\} .
$$

The following theorem is a variant of Hatori's local version of Bernard's Theorem:

Theorem 1. Let $B$ be a Banach function space on a compact Hausdorff space $X$, and let $x \in X$. If $l^{\infty}(\mathbf{N}, B) \mid \mathscr{F}_{x}$ is dense in $C\left(\mathscr{F}_{x}\right)$ then there exists a compact neighbourhood $K_{x}$ of $x$ such that $B \mid K_{x}=C\left(K_{x}\right)$.

Proof. Let $g \in C(X)$ be a function such that $\mathscr{F}_{x}=\mathscr{F}_{x}(g)$ and let $K_{n}=\left\{x^{\prime} \in\right.$ $\left.X:\left|g\left(x^{\prime}\right)-g(x)\right| \leq 1 / n\right\}$ for each $n \in \mathbf{N}$. We assert that there exists a natural number $n_{0}$ having the following property:

For all $f \in C(X)$ for which $\|f\|_{\infty} \leq 1$ there is a function $u \in B$ such that $N(u) \leq n_{0}$ and $|f-u|<1 / 2$ on $K_{n_{0}}$.

If not, then there is, for each $n \in \mathbf{N}$, a function $f_{n} \in C(X)$ such that $\left\|f_{n}\right\|_{\infty} \leq 1$ and such that $N(u)>n$ if $u \in B$ and $\left|f_{n}-u\right|<1 / 2$ on $K_{n}$. Since $\left\{f_{n}\right\} \in l^{\infty}(\mathbf{N}, C(X))$ there is a sequence $\left\{u_{n}\right\} \in l^{\infty}(\mathbf{N}, B)$ such that $\left|\left\{f_{n}\right\}-\left\{u_{n}\right\}\right|<1 / 2$ on $\mathscr{F}_{x}$ and hence on some neighbourhood $\mathscr{U}$ of $\mathscr{F}_{x}$. Since $\mathscr{U}$ is open the definition of $\mathscr{F}_{x}$ shows that there is a number $n_{0} \in \mathbf{N}$ such that

$$
\left\{\xi \in \beta(\mathbf{N} \times X):|\{g\}(\xi)-g(x)| \leq 1 / n_{0}\right\} \subseteq \mathscr{U} \text {. }
$$

Now, $\mathbf{N} \times K_{n_{0}}$ is contained in the set on the left hand side and hence in $\mathscr{U}$. We conclude that $\left|\left\{f_{n}\right\}-\left\{u_{n}\right\}\right|<1 / 2$ on $\mathbf{N} \times K_{n_{0}}$, which means that $\left|f_{n}-u_{n}\right|<1 / 2$ on $K_{n_{0}}$ for all $n$. Since $\left\{u_{n}\right\} \in l^{\infty}(\mathbf{N}, B)$ there is a number $n_{1}>n_{0}$ such that $N\left(u_{n}\right)<n_{1}$ for all $n$. Since $K_{n_{1}} \subseteq K_{n_{0}}$ we have reached a contradiction, and the assertion has been proved.

Let $f \in C(X)_{1}$, the unit ball of $C(X)$. By induction we can construct elements $v_{0}, v_{1}, \ldots, v_{n}, \ldots$ in $B$ such that $N\left(v_{n}\right)<n_{0}$ for all $n$ and such that

$$
\left|f-\left(v_{0}+\frac{1}{2} v_{1}+\cdots+\frac{1}{2^{n}} v_{n}\right)\right|<\frac{1}{2^{n+1}} \text { on } K_{n_{0}} .
$$

Let $v=\sum v_{n} / 2^{n}$. Then $v \in B$ and $v=f$ on $K_{n_{0}}$. 
Using this theorem, the Baire Category Theorem in the same way as Sidney in [6] and a few results from the theory of function algebras, we obtain a simple proof of the conjecture.

Theorem 2. ([1, 3, 6]). Let $A$ be a function algebra on a compact Hausdorff space $X$ and suppose there is a non-affine function defined on a subinterval of $\mathbf{R}$ which operates on $\operatorname{Re} A$. Then $A=C(X)$.

Proof. We have already noted that $\operatorname{Re} A$ is ultraseparating on $X$. Suppose $A \neq C(X)$, let $\mu \in \operatorname{ext}\left(\left(A^{\perp}\right)_{1}\right)$, and let $E$ be the smallest $p$-set for $A$ which contains the support of $\mu$. Then $u(E)$ is an interval for any $u \in \operatorname{Re} A$. To see this let $u \in \operatorname{Re} A$ and pick $a \in A$ such that $u=\operatorname{Re} a$. If $u(E)$ is not an interval, then we can find a real number $t$ such that $a(E)=C_{1} \cup C_{2}$, where $C_{1}$ and $C_{2}$ are nonempty disjoint compact subsets of the complex plane such that $\operatorname{Re} z<t$ for all $z \in C_{1}$ and $\operatorname{Re} z>t$ for all $z \in C_{2}$. By Runge's Theorem there is a sequence of polynomials converging uniformly to 0 on $C_{1}$ and to 1 on $C_{2}$. It follows that $E=a^{-1}\left(C_{1}\right) \cup a^{-1}\left(C_{2}\right)$, a union of two disjoint $p$ sets. Since $\mu$ is an extreme point of $\left(A^{\perp}\right)_{1}$, the support of $\mu$ is contained in $a^{-1}\left(C_{1}\right)$ or in $a^{-1}\left(C_{2}\right)$, contradicting the fact that $E$ is the smallest $p$-set for $A$ with this property.

We may assume that the operating function $h$ is defined on an interval containing 0 as an interior point and is not affine on any open interval which contains 0 . We choose a positive number $\delta$ such that $h \circ u$ is defined if $N(u) \leq \delta$, put $H=\{u \in \operatorname{Re} A: 0 \in u(E)$ and $N(u) \leq \delta\}$ and write $H=\cup H_{n}$, where $H_{n}=\{u \in H: N(h \circ u) \leq n\}$. By the Baire Category Theorem there is a number $n_{0} \in \mathbf{N}$ such that $\bar{H}_{n_{0}}$ contains a set which is open in $H$. (The bar denotes the closure w.r.t. the norm $N$.) Thus there is a function $u_{0} \in H$ and a positive number $\varepsilon$ such that $\left\{u \in H: N\left(u-u_{0}\right)<\varepsilon\right\} \subseteq \bar{H}_{n_{0}}$. Adding to or subtracting from $u_{0}$ a small multiple of the constant function 1, one obtains a function $u_{0}^{\prime}$ such that 0 is an interior point of $u_{0}^{\prime}(E)$. This assumes that $u_{0}$ is not constant on $E$. In that case we replace $u_{0}$ by another function $u$ in $H$ for which $N\left(u-u_{0}\right)<\varepsilon$ and $\varepsilon$ by a smaller number. Unless $E$ is a singleton, in which case we already have a contradiction, we can thus assume that 0 is an interior point of $u_{0}(E)$. Since $u(E)$ is an interval for all $u \in \operatorname{Re} A$ we conclude that $u \in H$ if $u$ is sufficiently close to $u_{0}$, and hence we can assume that

$$
\left\{u \in \operatorname{Re} A: N\left(u-u_{0}\right)<\varepsilon\right\} \subseteq \bar{H}_{n_{0}} .
$$

It follows that $N\left(h \circ\left(u_{0}+u\right)\right)<n_{0}$ if $u$ belongs to a dense subset of $\operatorname{Re} A_{\varepsilon}:=$ $\{u \in \operatorname{Re} A: N(u)<\varepsilon\}$ and thus $h \circ\left\{u_{0}+u_{n}\right\} \in l^{\infty}(\mathbf{N}, \operatorname{Re} A)$ if the functions $u_{n}$ belong to a dense subset of $\operatorname{Re} A_{\varepsilon}$. We conclude that $h \circ\left\{u_{0}+u_{n}\right\} \in \overline{l^{\infty}(\mathbf{N}, \operatorname{Re} A)}$ if the functions $u_{n}$ belong to $\operatorname{Re} A_{\varepsilon}$. (The bar denotes closure w.r.t. the supnorm on $\beta(\mathbf{N} \times X)$.)

We now pick a point $x \in E$ such that $u_{0}(x)=0$. Let $\mathscr{F}_{x}=\{\xi \in$ $\left.\beta(\mathbf{N} \times X):\left\{u_{0}\right\}(\xi)=u_{0}(x)\right\}$ and look at the space $l^{\infty}(\mathbf{N}, \operatorname{Re} A) \mid \mathscr{F}_{x}$. Since $\left\{u_{0}+u_{n}\right\}\left|\mathscr{F}_{x}=\left\{u_{n}\right\}\right| \mathscr{F}_{x}$, the proof of the theorem of de Leeuw and Katznel- 
son shows that $\left\{u_{n}\right\}^{2} \mid \mathscr{F}_{x} \in \overline{l^{\infty}(\mathbf{N}, \operatorname{Re} A) \mid \mathscr{F}_{x}}$ (where the closure is taken w.r.t. the supremum norm on $\mathscr{F}_{x}$ ) if the functions $u_{n}$ belong to $\operatorname{Re} A_{\varepsilon}$, and hence $\overline{l^{\infty}(\mathbf{N}, \operatorname{Re} A) \mid \mathscr{F}_{x}}=C\left(\mathscr{F}_{x}\right)$ by the Stone-Weierstrass Theorem.

By Theorem 1 there is a compact neighbourhood $K_{x}$ of $x$ such that $\operatorname{Re} A \mid K_{x}$ $=C\left(K_{x}\right)$. Using a theorem of S. Sidney and E. Stout [7] we infer that $A \mid K_{x}=$ $C\left(K_{x}\right)$. Since $A$ is a Dirichlet algebra, a theorem of I. Glicksberg [2] shows that $\nu \mid K_{x}=0$ for all $\nu \in A^{\perp}$, and thus $\nu \mid\left(X \backslash U_{x}\right) \in A^{\perp}$ for all $\nu \in A^{\perp}$, where $U_{x}$ is the interior of $K_{x}$. Referring again to a theorem of I. Glicksberg [2], the set $X \backslash U_{x}$ is a $p$-set for $A$ supporting $\mu$, hence $E \backslash U_{x}$ is a $p$-set which supports $\mu$, contradicting the fact that $E$ is the smallest $p$-set with this property. This concludes the proof.

\section{REFERENCES}

1. A. Bernard, Espaces des parties réelles des éléments d'une algebre de Banach de fonctions, J. Funct. Anal. 10 (1972), 387-409.

2. I. Glicksberg, Measures orthogonal to algebras and sets of antisymmetry, Trans. Amer. Math. Soc. 105 (1962), 415-435.

3. O. Hatori, Functions which operate on the real part of a uniform algebra, Proc. Amer. Math. Soc. 83 (1981), 565-568.

4. __ Range transformations on a Banach function algebra II, Pacific J. Math. 138 89-118.

5. K. de Leeuw and Y. Katznelson, Functions that operate on non-selfadjoint algebras, J. Analyse Math. 11 (1963), 207-219.

6. S. J. Sidney, Functions which operate on the real part of a uniform algebra, Pacific J. Math. 80 (1979), 265-272.

7. S. J. Sidney and E. L. Stout, A note on interpolation. Proc. Amer. Math. Soc. 19 (1968), 380-382.

8. J. Wermer, The space of real parts of a function algebra, Pacific J. Math. 13 (1963), 14231426 .

Science Institute, University of Iceland, 3 Dunhaga, Reykjavik, Iceland 